\title{
Inhaltsverzeichnis
}

Seite

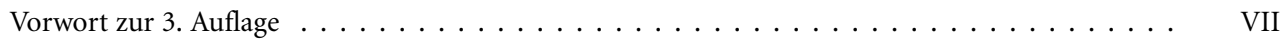

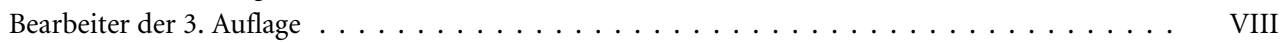

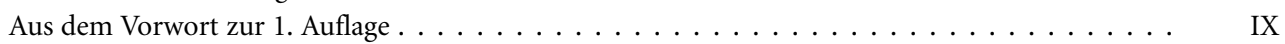

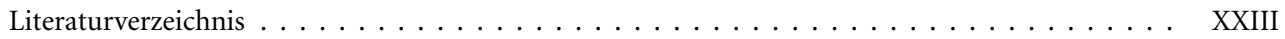

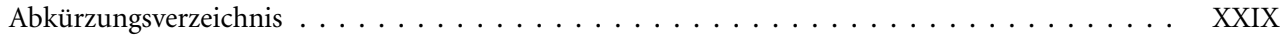

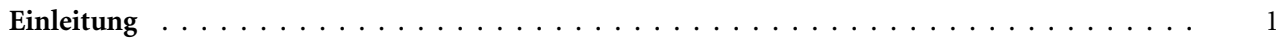

Grundgesetz Art. $12 \ldots \ldots \ldots \ldots \ldots \ldots \ldots \ldots \ldots \ldots$

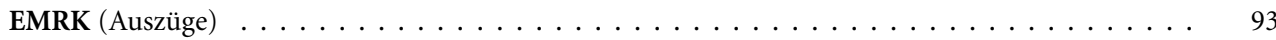

Bundesrechtsanwaltsordnung (BRAO) mit korrespondierenden BORA-Vorschriften

$\$ 1$ Stellung des Rechtsanwalts in der Rechtspflege . . . . . . . . . . . . . . . . . . . . 171

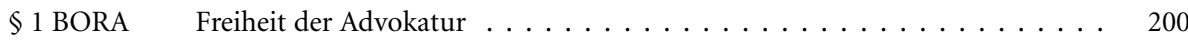

$\S 18$ BORA Vermittelnde, schlichtende oder mediative Tätigkeit . . . . . . . . . . 203

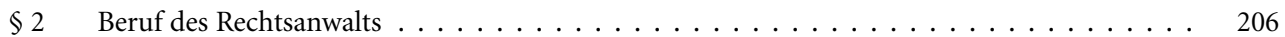

$\S 26$ BORA Beschäftigung von Rechtsanwälten und anderen Mitarbeitern . . . . . . . . 225

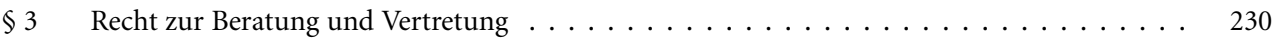

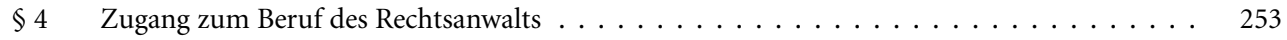

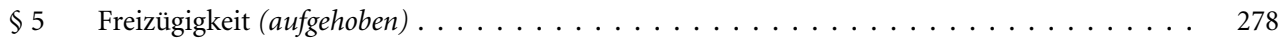

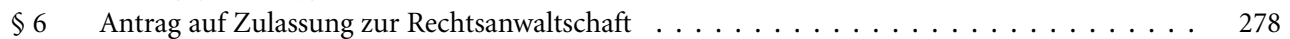

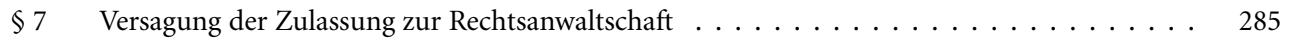

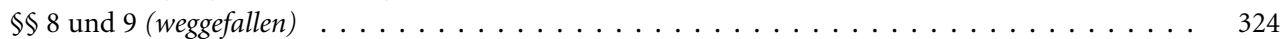

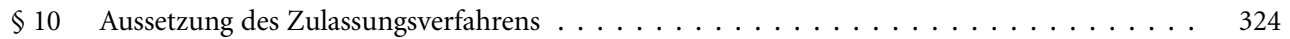

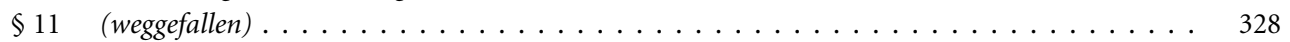

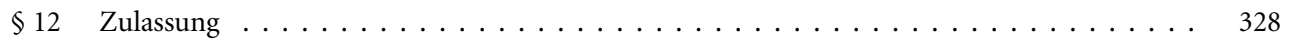

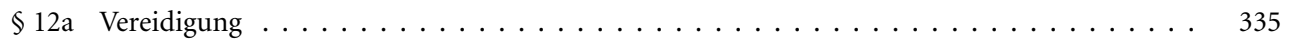

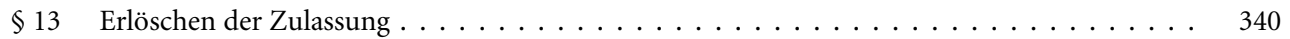

$\$ 14$ Rücknahme und Widerruf der Zulassung . . . . . . . . . . . . . . . . . . . . . . . . . . 343

$\S 15$ Ärztliches Gutachten bei Versagung und Widerruf der Zulassung . . . . . . . . . . . . . . . 366

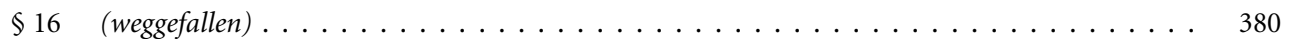

$\S 17$ Erlöschen der Befugnis zur Führung der Berufsbezeichnung . . . . . . . . . . . . . . . 380

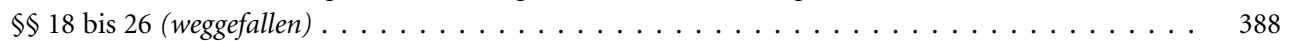

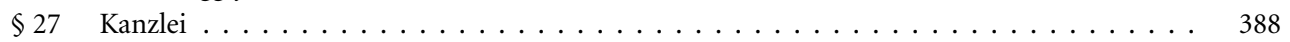

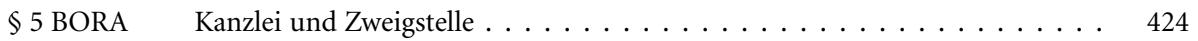

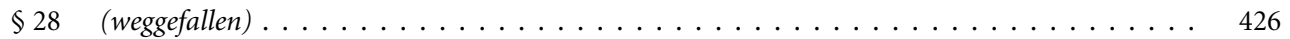

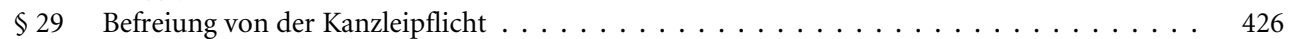

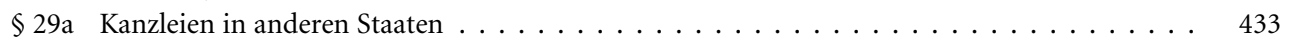

$\$ 30$ Zustellungsbevollmächtigter. . . . . . . . . . . . . . . . . . . . . . . . . 441

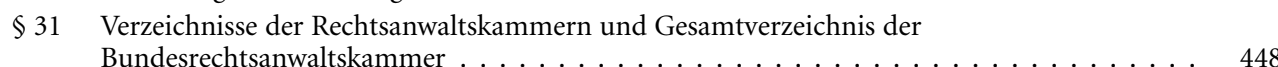

$\S 31$ a Besonderes elektronisches Anwaltspostfach . . . . . . . . . . . . . . . . . . 471

$\S 31$ b Europäisches Rechtsanwaltsverzeichnis . . . . . . . . . . . . . . . . . . . . . . . 489

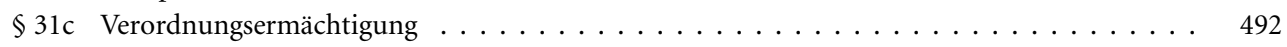


Seite

$\S 32$ Ergänzende Anwendung des Verwaltungsverfahrensgesetzes . . . . . . . . . . . . . . . . . . 529

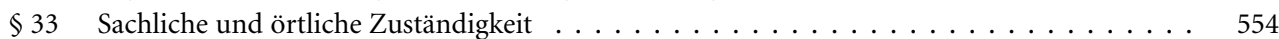

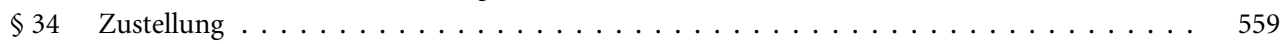

$\S 35$ Bestellung eines Vertreters im Verwaltungsverfahren . . . . . . . . . . . . . . . . . . 561

$\S 36$ Ermittlung des Sachverhalts und Übermittlung personenbezogener Daten . . . . . . . . . . . . . . . . . . . . . . . . . . . . . . . . . . . . .

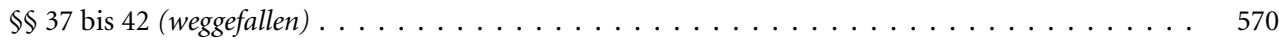

Vorbemerkung Vor $\$ \$ 43 \mathrm{ff} . \ldots \ldots \ldots \ldots \ldots \ldots \ldots$

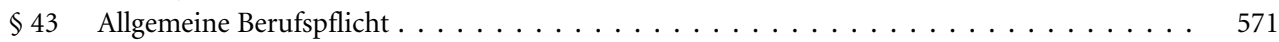

$\$ 11$ BORA Unterrichtung des Mandanten . . . . . . . . . . . . . . . . . . . 591

$\S 12$ BORA $\quad$ Umgehung des Gegenanwalts . . . . . . . . . . . . . . . . . . . . . 600

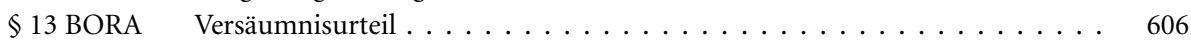

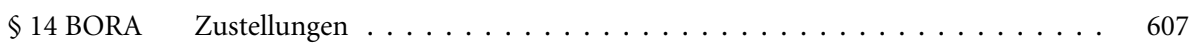

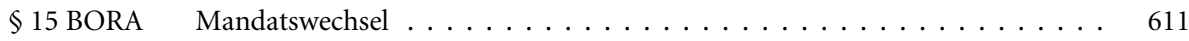

$\S 16$ BORA $\quad$ Prozesskostenhilfe und Beratungshilfe . . . . . . . . . . . . . . . . . . . . . 614

$\S 16$ a BORA Ablehnung der Beratungshilfe . . . . . . . . . . . . . . . . . . 619

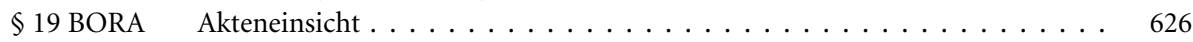

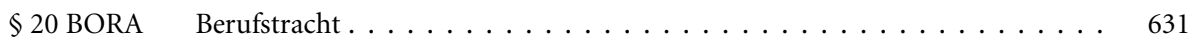

$\S 25$ BORA Beanstandungen gegenüber Kollegen $\ldots \ldots \ldots \ldots \ldots$

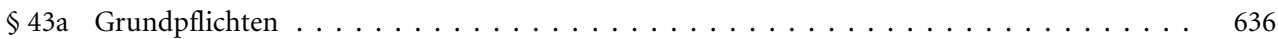

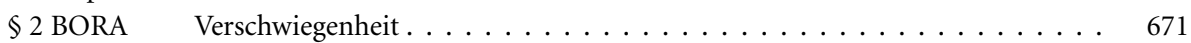

$\S 3$ BORA $\quad$ Widerstreitende Interessen, Versagung der Berufstätigkeit . . . . . . . . . . . . 688

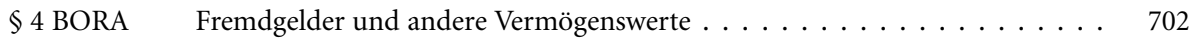

$\S 43 \mathrm{~b}$ Werbung $\ldots \ldots \ldots \ldots \ldots \ldots \ldots \ldots$

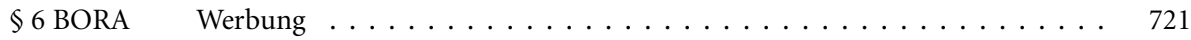

$\S 7$ BORA Benennung von Teilbereichen der Berufstätigkeit . . . . . . . . . . . 722

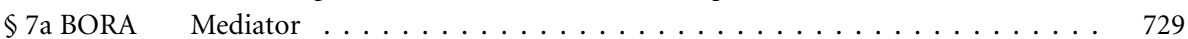

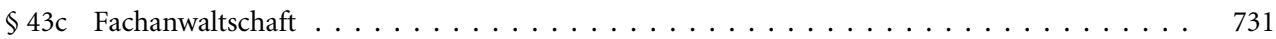

$\S 43 \mathrm{~d}$ Darlegungs- und Informationspflichten bei Inkassodienstleistungen . . . . . . . . . . . . 744

$\$ 43 \mathrm{e}$ Inanspruchnahme von Dienstleistungen $\ldots \ldots \ldots \ldots \ldots \ldots \ldots \ldots \ldots$

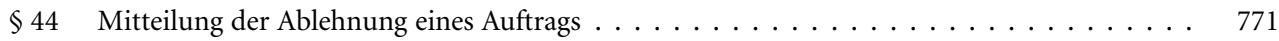

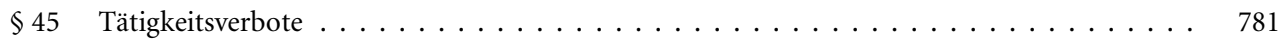

$\S 46$ Angestellte Rechtsanwälte und Syndikusrechtsanwälte . . . . . . . . . . . . . . . . 801

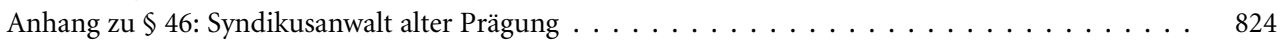

$\$ 46 \mathrm{a}$ Zulassung als Syndikusrechtsanwalt . . . . . . . . . . . . . . . . . . . . . . . . 829

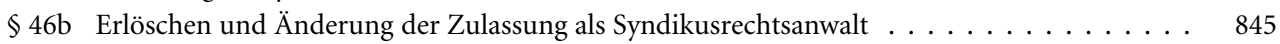

$\S 46$ c Besondere Vorschriften für Syndikusrechtsanwälte $\ldots \ldots \ldots \ldots \ldots \ldots$

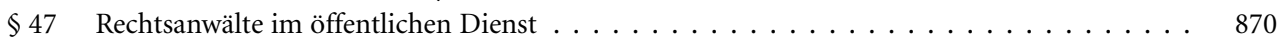

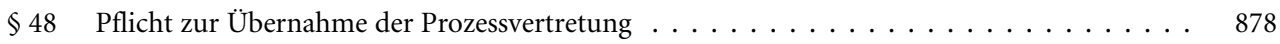

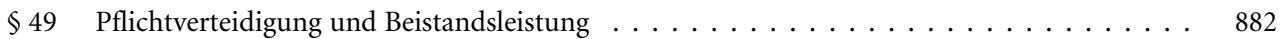

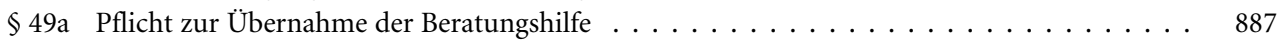

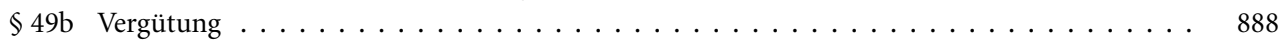

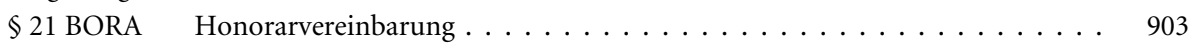

$\$ 22$ BORA Gebühren- und Honorarteilung . . . . . . . . . . . . . . . . . . . . . . . . . . 904

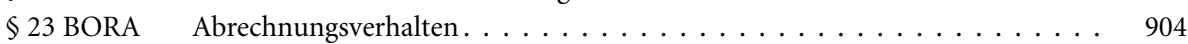

$\$ 49 \mathrm{c}$ Einreichung von $\mathrm{Schutzschriften} \ldots \ldots \ldots \ldots \ldots \ldots \ldots \ldots \ldots \ldots$

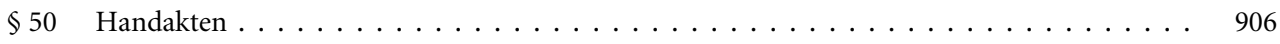

$\S 17$ BORA Zurückbehaltung von Handakten . . . . . . . . . . . . . . . . . . . . . . . . . . . . . . . . . . .

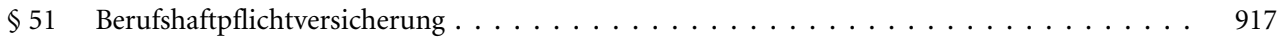


\$51a Berufshaftpflichtversicherung einer Partnerschaftsgesellschaft mit beschränkter

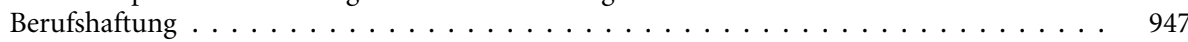

$\S 52$ Vertragliche Begrenzung von Ersatzansprüchen . . . . . . . . . . . . . . . . . . . . . . . . . . . . . . . . . . . . . . .

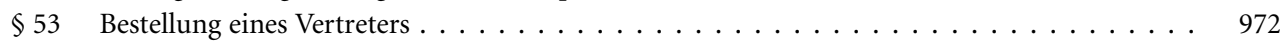

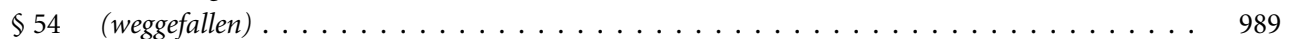

$\S 55$ Bestellung eines Abwicklers der Kanzlei . . . . . . . . . . . . . . . . . . . . . . . . . . . . . . . . . . . . . . .

$\S 56$ Besondere Pflichten gegenüber dem Vorstand der Rechtsanwaltskammer . . . . . . . . . . . 1015

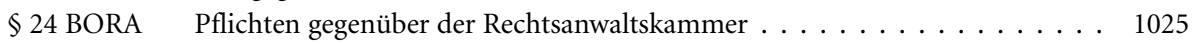

$\$ 57$ Zwangsgeld bei Verletzung der besonderen Pflichten . . . . . . . . . . . . . . . . . . . . . 1029

$\S 58$ Einsicht in die Personalakten . . . . . . . . . . . . . . . . . . . . . . . . . . 1034

$\S 59$ Ausbildung von Referendaren . . . . . . . . . . . . . . . . . . . . . . . 1038

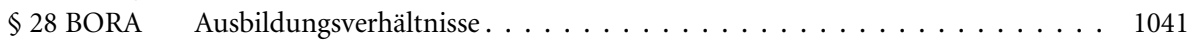

$\S 59$ a Berufliche Zusammenarbeit . . . . . . . . . . . . . . . . . . . . . . . . . . . 1044

$\$ 8$ BORA Kundgabe gemeinschaftlicher Berufsausübung und anderer beruflicher

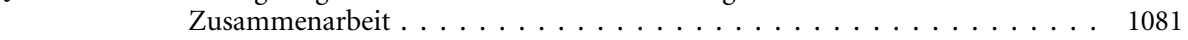

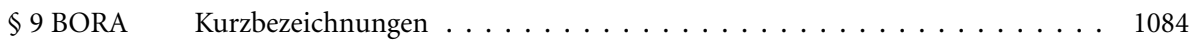

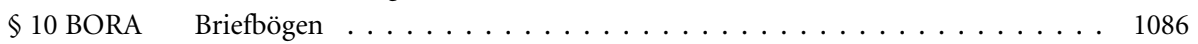

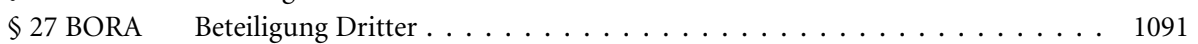

$\S 30$ BORA Berufliche Zusammenarbeit mit Angehörigen anderer Berufe . . . . . . . . 1092

$\S 31$ BORA $\quad$ (ehedem Verbot der Sternsozietät, aufgehoben) . . . . . . . . . . . . . . . 1097

$\S 32$ BORA Beendigung einer gemeinschaftlichen Berufsausübung . . . . . . . . . . . . . . 1097

$\S 33$ BORA Geltung der Berufsordnung bei beruflicher Zusammenarbeit . . . . . . . . . 1104

$\S 59 \mathrm{~b}$ Satzungskompetenz . . . . . . . . . . . . . . . . . . . . . . . 1108

\$59c Zulassung als Rechtsanwaltsgesellschaft, Beteiligung an beruflichen Zusammenschlüssen . . . 1118

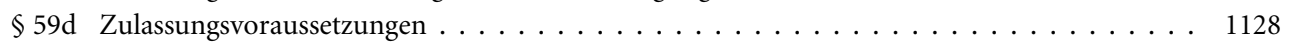

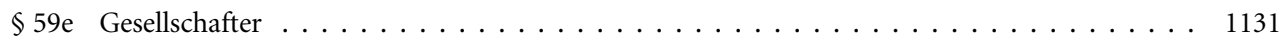

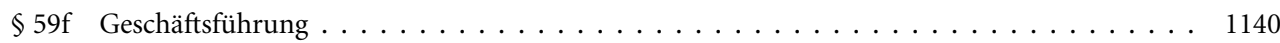

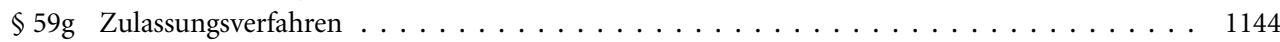

$\S 59$ h Erlöschen der Zulassung . . . . . . . . . . . . . . . . . . . . . . . . 1146

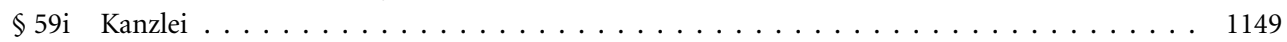

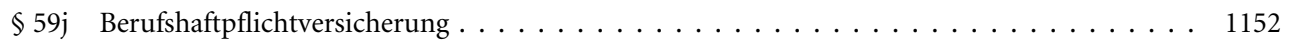

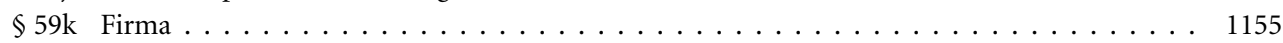

$\S 591$ Vertretung vor Gerichten und Behörden _ . . . . . . . . . . . . . . . . . . . 1156

$\S 59 \mathrm{~m}$ Mitteilungspflichten, anwendbare Vorschriften und Verschwiegenheitspflicht $\ldots \ldots \ldots$

$\$ 60$ Bildung und Zusammensetzung der Rechtsanwaltskammer . . . . . . . . . . . . . . . . . . . . . . . . . . . . . . . . .

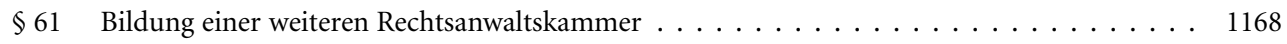

$\$ 62$ Stellung der Rechtsanwaltskammer . . . . . . . . . . . . . . . . . . . . . 1172

$\$ 63$ Zusammensetzung des Vorstandes . . . . . . . . . . . . . . . . . . . . . . . 1182

$\$ 64$ Wahlen zum Vorstand . . . . . . . . . . . . . . . . . . . . . . . . . 1185

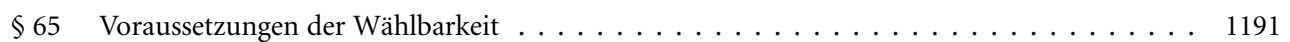

$\$ 66$ Ausschluss von der Wählbarkeit . . . . . . . . . . . . . . . . . . . . . . . . . . 1194

$\$ 67$ Recht zur Ablehnung der Wahl . . . . . . . . . . . . . . . . . . . . . . . . . 1196

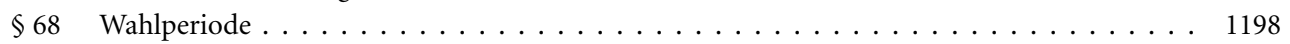

$\$ 69$ Vorzeitiges Ausscheiden eines Vorstandsmitgliedes . . . . . . . . . . . . . . . . . . 1200

$\$ 70$ Sitzungen des Vorstandes . . . . . . . . . . . . . . . . . . . . . . . . . . 1204

$\$ 71$ Beschlussfähigkeit des Vorstandes . . . . . . . . . . . . . . . . . . . . . . . . 1206

$\$ 72$ Beschlüsse des Vorstandes . . . . . . . . . . . . . . . . . . . . . . . . . . . . . . . 1207

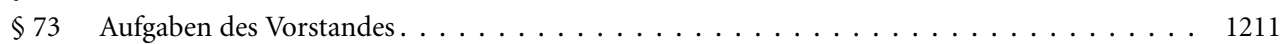


Seite

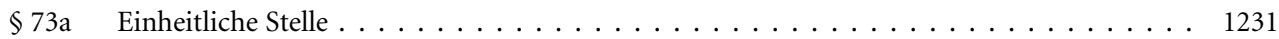

$\S 73 \mathrm{~b} \quad$ Verwaltungsbehörde für Ordnungswidrigkeiten . . . . . . . . . . . . . . . . 1233

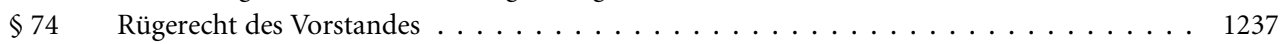

$\S 74 \mathrm{a} \quad$ Antrag auf anwaltsgerichtliche Entscheidung . . . . . . . . . . . . . . . . . . . 1252

$\$ 75 \quad$ Ehrenamtliche Tätigkeit des Vorstandes . . . . . . . . . . . . . . . . . . . . . . . . . 1258

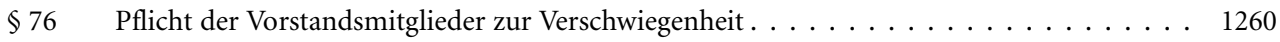

$\$ 77 \quad$ Abteilungen des Vorstandes . . . . . . . . . . . . . . . . . . . . . . . . . . 1270

$\S 78 \quad$ Zusammensetzung und Wahl des Präsidiums . . . . . . . . . . . . . . . . . . . 1274

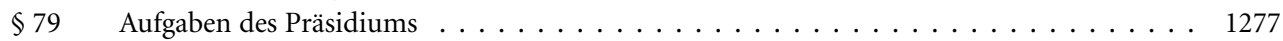

$\$ 80 \quad$ Aufgaben des Präsidenten . . . . . . . . . . . . . . . . . . . . . . . . . . . 1279

$\S 81$ Berichte über die Tätigkeit der Kammer und über Wahlergebnisse . . . . . . . . . . . . . 1281

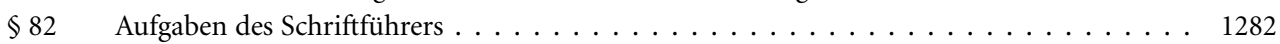

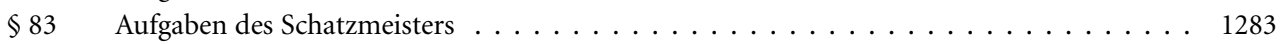

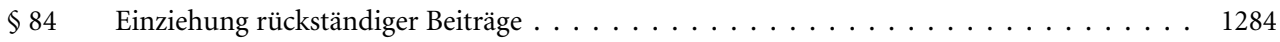

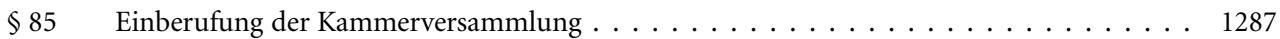

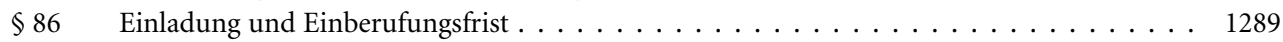

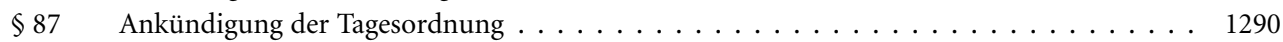

$\S 88 \quad$ Wahlen und Beschlüsse der Kammer . . . . . . . . . . . . . . . . . . . . . . . . . 1291

$\$ 89$ Aufgaben der Kammerversammlung . . . . . . . . . . . . . . . . . . . . . . . . . . 1294

$\$ \$ 90,91$ (weggefallen) $\ldots \ldots \ldots \ldots \ldots \ldots \ldots \ldots \ldots \ldots \ldots$

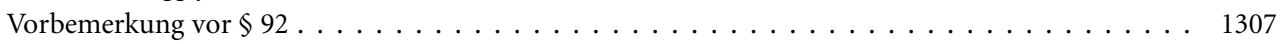

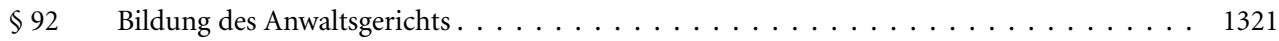

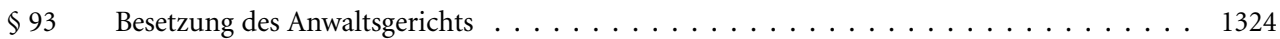

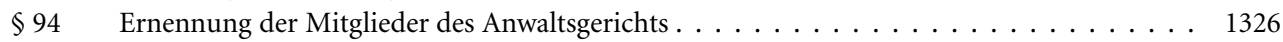

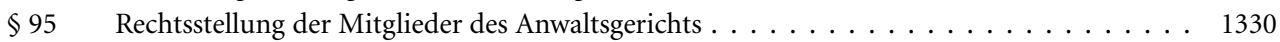

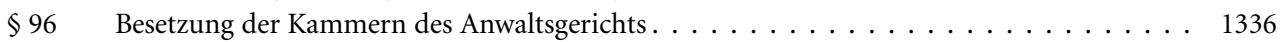

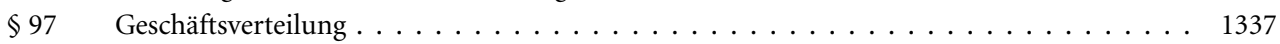

$\$ 98 \quad$ Geschäftsstelle und Geschäftsordnung . . . . . . . . . . . . . . . . . . . . . 1338

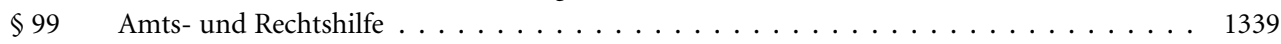

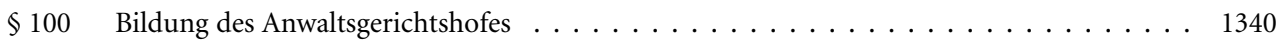

$\$ 101$ Besetzung des Anwaltsgerichtshofes . . . . . . . . . . . . . . . . . . . . . . . 1342

$\$ 102$ Bestellung von Berufsrichtern zu Mitgliedern des Anwaltsgerichtshofes . . . . . . . . . . . . 1344

$\$ 103$ Ernennung von Rechtsanwälten zu Mitgliedern des Anwaltsgerichtshofes . . . . . . . . . . . 1346

$\$ 104$ Besetzung der Senate des Anwaltsgerichtshofes . . . . . . . . . . . . . . . . . . . . . 1348

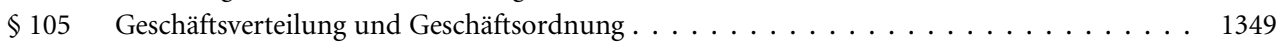

$\$ 106$ Besetzung des Senats für Anwaltssachen . . . . . . . . . . . . . . . . . . . . . . . . 1349

$\$ 107$ Rechtsanwälte als Beisitzer . . . . . . . . . . . . . . . . . . . . . . . 1352

$\S 108$ Voraussetzungen für die Berufung zum Beisitzer und Recht zur Ablehnung . . . . . . . . . . 1354

$\$ 109$ Beendigung des Amtes als Beisitzer . . . . . . . . . . . . . . . . . . . . . . . . 1354

$\S 110$ Stellung der Rechtsanwälte als Beisitzer und Pflicht zur Verschwiegenheit . . . . . . . . . . 1356

$\$ 111$ Reihenfolge der Teilnahme an den Sitzungen . . . . . . . . . . . . . . . . . . . . . . . . . . . . . . . . . . . . . . . . . . . . . .

$\$ 112$ Entschädigung der anwaltlichen Beisitzer . . . . . . . . . . . . . . . . . . . . . 1359

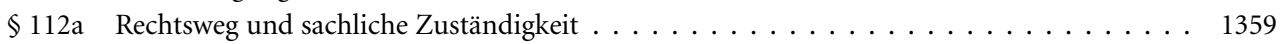

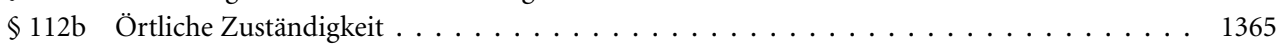

$\S 112$ c Anwendung der Verwaltungsgerichtsordnung . . . . . . . . . . . . . . . . . . . . . . . . . . . . . . . . . . . . . . . . . . . . . . .

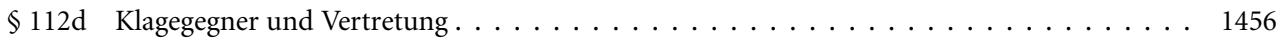

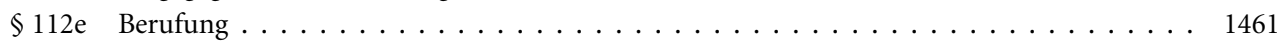

$\S 112 \mathrm{f}$ Klagen gegen Wahlen und Beschlüsse . . . . . . . . . . . . . . . . . . . . . . . 1496 
Seite

$\$ 112 \mathrm{~g} \quad$ Rechtsschutz bei überlangen Gerichtsverfahren $\ldots \ldots \ldots \ldots \ldots$. . . . . . . . . . . . . . 1503

$\S 112 \mathrm{~h}$ Verwendung gefälschter Berufsqualifikationsnachweise . . . . . . . . . . . . . 1513

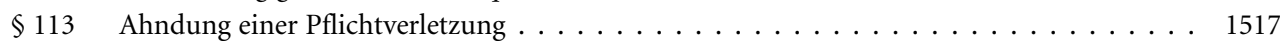

$\$ 114$ Anwaltsgerichtliche Maßnahmen . . . . . . . . . . . . . . . . . . . . . 1530

$\$ 114 \mathrm{a}$ Wirkungen des Vertretungsverbots, Zuwiderhandlungen . . . . . . . . . . . . . . . . . . . . . . . . . . . . . . . . . . . . . . . . . . . . .

$\$ 115$ Verjährung der Verfolgung einer Pflichtverletzung . . . . . . . . . . . . . . . . . . . 1539

$\S 115$ a Rüge und anwaltsgerichtliche Maßnahme . . . . . . . . . . . . . . . . . . . . . . . 1541

$\$ 115 \mathrm{~b}$ Anderweitige Ahndung . . . . . . . . . . . . . . . . . . . . . . . . . . . 1544

$\$ 115$ c Vorschriften für Geschäftsführer von Rechtsanwaltsgesellschaften . . . . . . . . . . . . . 1548

$\$ 116$ Vorschriften für das Verfahren und den Rechtsschutz bei überlangen Gerichtsverfahren $\ldots 1550$

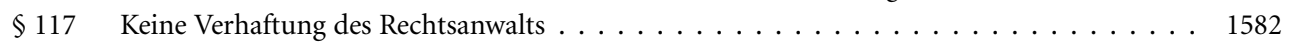

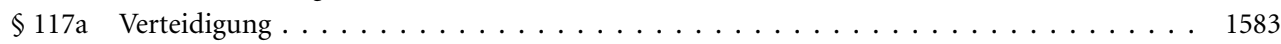

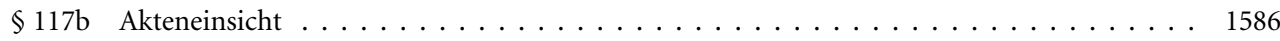

$\$ 118$ Verhältnis des anwaltsgerichtlichen Verfahrens zum Straf- oder Bußgeldverfahren . . . . . 1588

$\S 118$ a Verhältnis des anwaltsgerichtlichen Verfahrens zu dem Verfahren anderer

Berufsgerichtsbarkeiten ... . . . . . . . . . . . . . . . . . . . 1600

$\$ 118$ b Aussetzung des anwaltsgerichtlichen Verfahrens . . . . . . . . . . . . . . . . . . . . . . . . . . . . . . . . . . . . . . . . . . . . . . . . . . . . . .

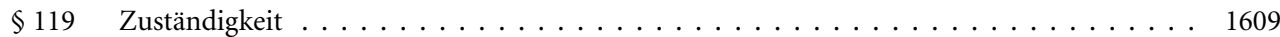

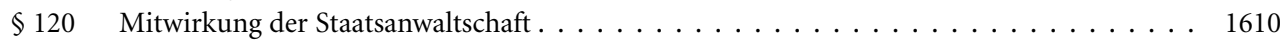

$\$ 120 \mathrm{a}$ Gegenseitige Unterrichtung von Staatsanwaltschaft und Rechtsanwaltskammern . . . . . . 1612

$\$ 121$ Einleitung des anwaltsgerichtlichen Verfahrens . . . . . . . . . . . . . . . . . . . 1613

$\$ 122$ Gerichtliche Entscheidung über die Einleitung des Verfahrens . . . . . . . . . . . . . . . . . 1614

$\$ 123$ Antrag des Rechtsanwalts auf Einleitung des anwaltsgerichtlichen Verfahrens . . . . . . . 1620

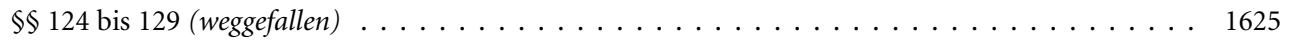

$\$ 130 \quad$ Inhalt der Anschuldigungsschrift $\ldots \ldots \ldots \ldots \ldots \ldots$

$\$ 131$ Entscheidung über die Eröffnung des Hauptverfahrens vor dem Anwaltsgericht . . . . . . 1627

$\$ 132$ Rechtskraftwirkung eines ablehnenden Beschlusses . . . . . . . . . . . . . . . 1630

$\S 133$ Zustellung des Eröffnungsbeschlusses . . . . . . . . . . . . . . . . . . . . 1632

$\S 134$ Hauptverhandlung trotz Ausbleibens des Rechtsanwalts . . . . . . . . . . . . . . . . . . 1634

$\$ 135$ Nichtöffentliche Hauptverhandlung . . . . . . . . . . . . . . . . . . . . 1638

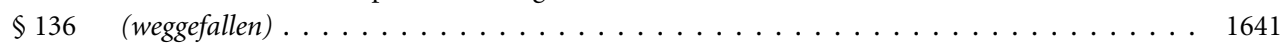

$\S 137$ Beweisaufnahme durch einen beauftragten oder ersuchten Richter . . . . . . . . . . . . 1641

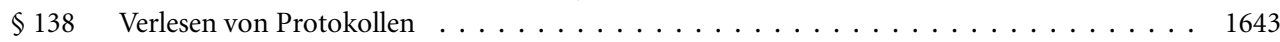

$\$ 139$ Entscheidung des Anwaltsgerichts . . . . . . . . . . . . . . . . . . . . . . . . 1647

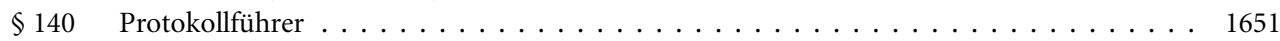

$\$ 141$ Ausfertigung der Entscheidungen $\ldots \ldots \ldots \ldots \ldots \ldots$

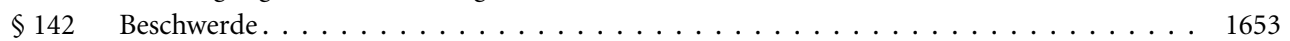

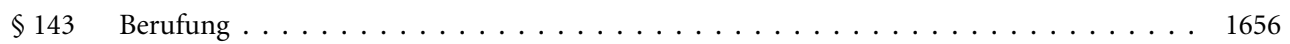

$\$ 144$ Mitwirkung der Staatsanwaltschaft vor dem Anwaltsgerichtshof $\ldots \ldots \ldots$. . . . . . . . 1667

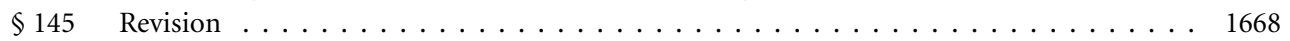

$\$ 146$ Einlegung der Revision und Verfahren . . . . . . . . . . . . . . . . . . . . . . . . . . . . . . . . . . . . .

$\$ 147$ Mitwirkung der Staatsanwaltschaft vor dem Bundesgerichtshof . . . . . . . . . . . . . 1682

$\$ 148$ Anordnung der Beweissicherung . . . . . . . . . . . . . . . . . . . . . . . . 1682

$\$ 149$ Verfahren . . . . . . . . . . . . . . . . . . . . . . . . . . . 1684

$\$ 150 \quad$ Voraussetzung für das Verbot $\ldots \ldots \ldots \ldots \ldots$

$\$ 150$ a Verfahren zur Erzwingung des Antrags der Staatsanwaltschaft . . . . . . . . . . . . . . 1693

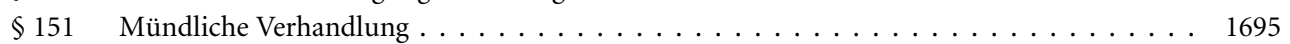

$\S 152$ Abstimmung über das Verbot . . . . . . . . . . . . . . . . . . . . . . . 1698 


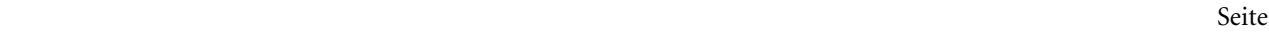

$\S 153 \quad$ Verbot im Anschluss an die Hauptverhandlung . . . . . . . . . . . . . . . . . . . 1698

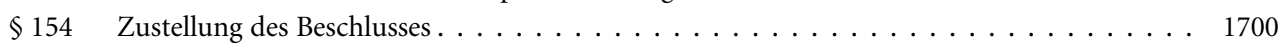

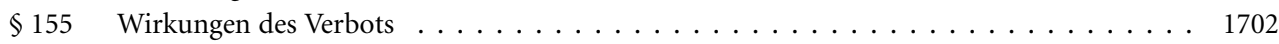

$\S 156$ Zuwiderhandlungen gegen das Verbot . . . . . . . . . . . . . . . . . . . . 1707

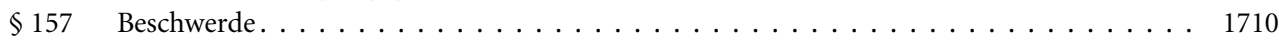

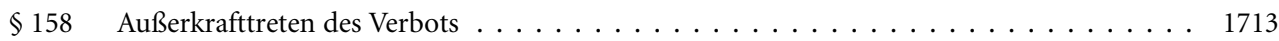

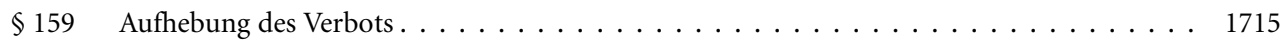

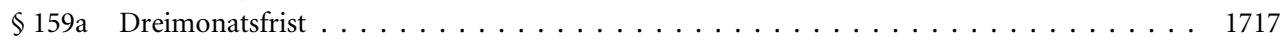

$\$ 159 \mathrm{~b}$ Prüfung der Fortdauer des Verbots . . . . . . . . . . . . . . . . . . . . . . . . . . 1720

$\$ 160 \quad$ Mitteilung des Verbots . . . . . . . . . . . . . . . . . . . . . . . . . . . . . 1722

$\$ 161 \quad$ Bestellung eines Vertreters . . . . . . . . . . . . . . . . . . . . . . . . . . . . . . . . 1724

$\S 161 \mathrm{a}$ Gegenständlich beschränktes Vertretungsverbot . . . . . . . . . . . . . . . . . . . . 1727

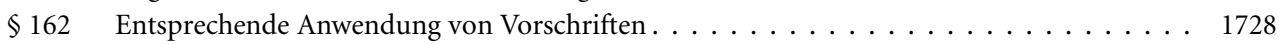

$\$ 163$ Sachliche Zuständigkeit . . . . . . . . . . . . . . . . . . . . . . . . . . . . 1729

$\$ 164$ Besondere Voraussetzung für die Zulassung . . . . . . . . . . . . . . . . . . . . 1738

$\$ 165$ Wahlausschuss für Rechtsanwälte bei dem Bundesgerichtshof . . . . . . . . . . . . . . 1750

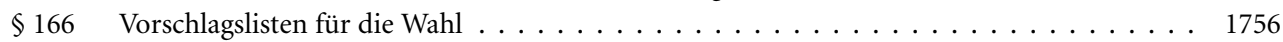

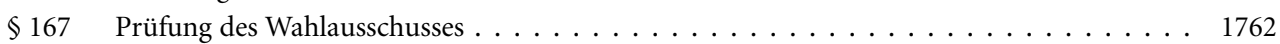

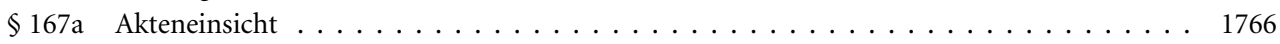

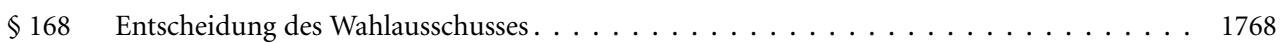

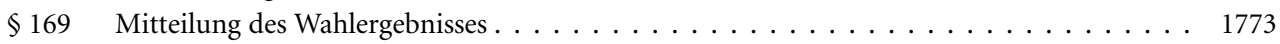

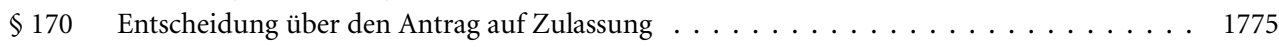

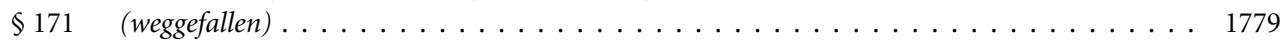

$\$ 172$ Beschränkung des Auftretens vor anderen Gerichten . . . . . . . . . . . . . . . . . . . . 1779

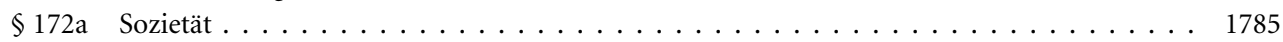

$\$ 172 \mathrm{~b}$ Kanzlei . . . . . . . . . . . . . . . . . . . . . . . . . . . . . 1789

$\S 173$ Bestellung eines Vertreters und eines Abwicklers der Kanzlei . . . . . . . . . . . . . . . . . 1790

$\$ 174$ Zusammensetzung und Vorstand . . . . . . . . . . . . . . . . . . . . . 1793

$\S 175$ Zusammensetzung und Sitz der Bundesrechtsanwaltskammer . . . . . . . . . . . . . 1796

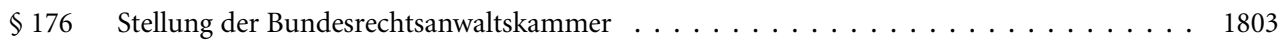

$\$ 177$ Aufgaben der Bundesrechtsanwaltskammer . . . . . . . . . . . . . . . . . . . . 1806

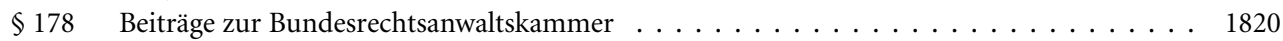

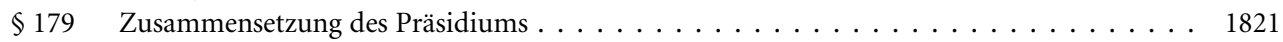

$\$ 180$ Wahlen zum Präsidium $\ldots \ldots \ldots \ldots \ldots \ldots \ldots \ldots \ldots \ldots \ldots \ldots$

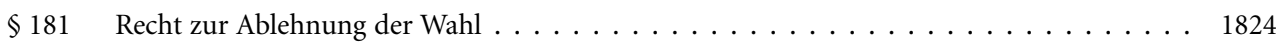

$\$ 182$ Wahlperiode und vorzeitiges Ausscheiden $\ldots \ldots \ldots \ldots \ldots \ldots \ldots$

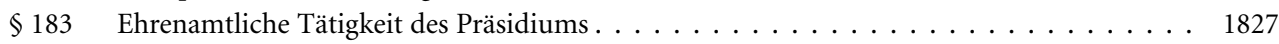

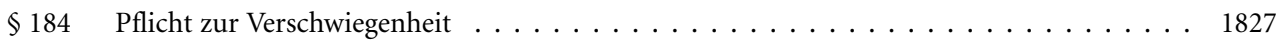

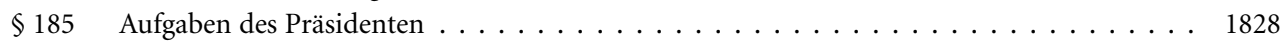

$\$ 186$ Aufgaben des Schatzmeisters . . . . . . . . . . . . . . . . . . . . . . . . . . . . . 1829

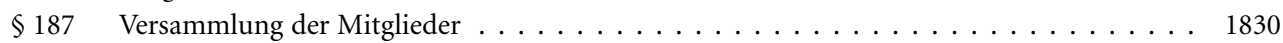

$\S 188$ Vertreter der Rechtsanwaltskammer in der Hauptversammlung . . . . . . . . . . . . . . 1831

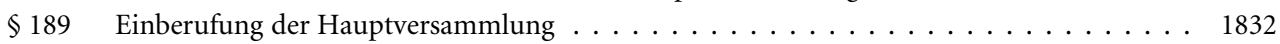

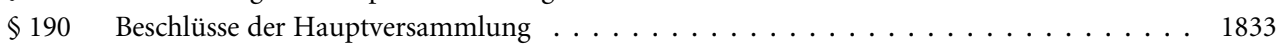

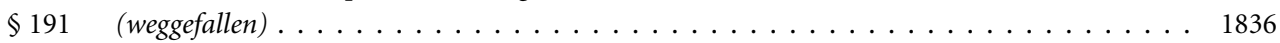

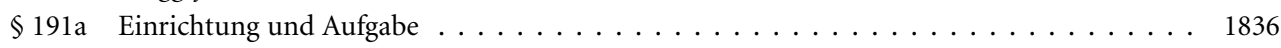

$\S 191$ b Wahl der stimmberechtigten Mitglieder der Satzungsversammlung . . . . . . . . . . . . 1841

$\S 191 \mathrm{c}$ Einberufung und Stimmrecht . . . . . . . . . . . . . . . . . . . . . . . 1845 
Seite
S. . . . . . . . . . . . . . . 1846

$\$ 191$ Leitung der Versammlung, Beschlussfassung . . . . . . . . . . . . . . . . . . . . . . . 1846

$\$ 191$ e Prüfung von Beschlüssen der Satzungsversammlung durch die Aufsichtsbehörde . . . . . 1848

$\$ 191 \mathrm{f}$ Schlichtungsstelle der Rechtsanwaltschaft . . . . . . . . . . . . . . . . . . . . . . . . . . . . . . . . . . . . . . . . . . . . .

$\$ 192$ Erhebung von Gebühren und Auslagen . . . . . . . . . . . . . . . . . . . . . . . . 1862

$\$ 193$ Gerichtskosten . . . . . . . . . . . . . . . . . . . . . . . . . 1864

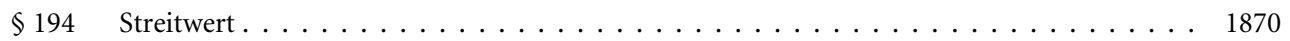

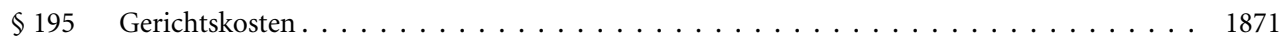

$\$ 196$ Kosten bei Anträgen auf Einleitung des anwaltsgerichtlichen Verfahrens . . . . . . . . . . 1874

$\$ 197$ Kostenpflicht des Verurteilten . . . . . . . . . . . . . . . . . . . . . . . . . . 1875

$\$ 197$ a Kostenpflicht in dem Verfahren bei Anträgen auf anwaltsgerichtliche Entscheidung gegen die Androhung oder die Festsetzung des Zwangsgelds oder über die Rüge . . . . . . . 1877

$\$ 198$ Haftung der Rechtsanwaltskammer . . . . . . . . . . . . . . . . . . . . 1878

$\$ 199$ Festsetzung der Kosten des Verfahrens vor dem Anwaltsgericht . . . . . . . . . . . . . . 1879

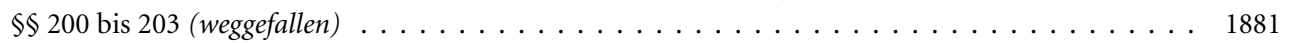

$\$ 204$ Vollstreckung anwaltsgerichtlicher Maßnahmen . . . . . . . . . . . . . . . 1881

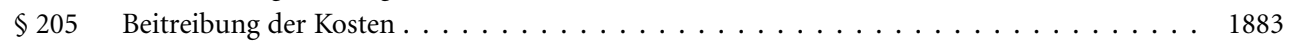

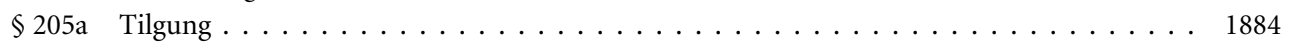

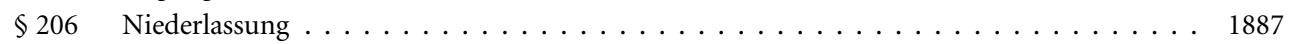

$\$ 207$ Aufnahmeverfahren und berufliche Stellung . . . . . . . . . . . . . . . . . . . 1901

$\$ 208$ Landesrechtliche Beschränkungen der Parteivertretung und Beistandschaft . . . . . . . . . 1905

$\$ 209$ Kammermitgliedschaft von Inhabern einer Erlaubnis nach dem Rechtsberatungsgesetz . . . 1907

$\$ 210$ Bestehenbleiben von Rechtsanwaltskammern . . . . . . . . . . . . . . . . . . . 1916

$\$ 211$ Befreiung von der Voraussetzung der Befähigung zum Richteramt . . . . . . . . . . . . 1917

$\$ 215$ Übergangsregelungen (aufgehoben) $\ldots \ldots \ldots \ldots \ldots$

\section{Übersicht BORA-Vorschriften}

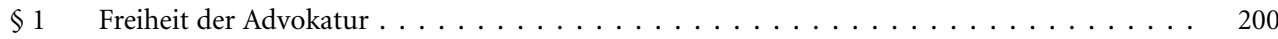

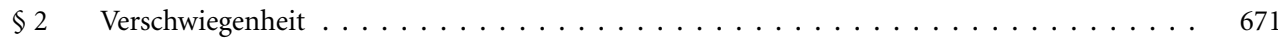

$\S 3$ Widerstreitende Interessen, Versagung der Berufstätigkeit . . . . . . . . . . . . . . 688

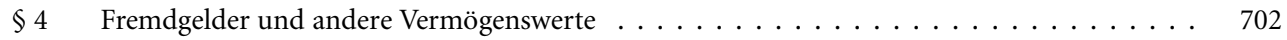

$\S 5 \quad$ Kanzlei und Zweigstelle . . . . . . . . . . . . . . . . . . . . . . . . . . 424

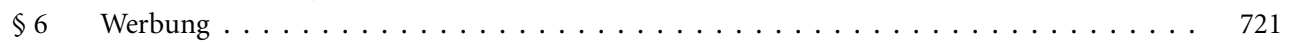

$\S 7$ Benennung von Teilbereichen der Berufstätigkeit . . . . . . . . . . . . . . . . 722

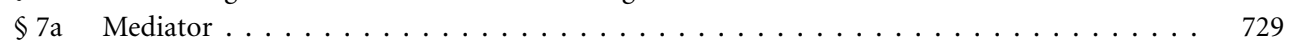

$\$ 8 \quad$ Kundgabe gemeinschaftlicher Berufsausübung und anderer beruflicher Zusammenarbeit . . 1081

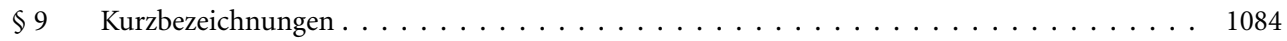

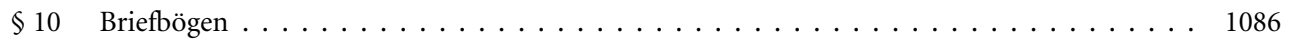

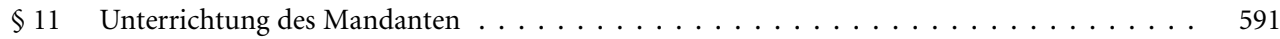

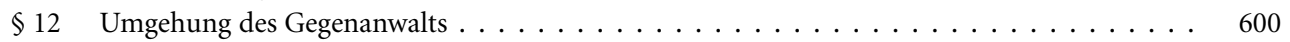

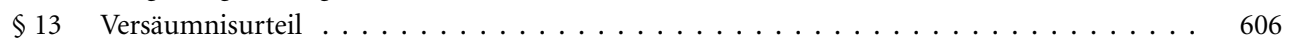

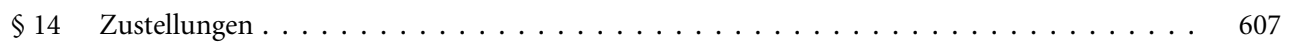

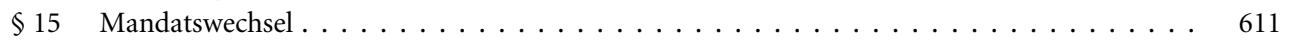

$\$ 16$ Prozesskostenhilfe und Beratungshilfe . . . . . . . . . . . . . . . . . . . . . . . . . . . . . . . . . . . . .

$\$ 16$ a Ablehnung der Beratungshilfe . . . . . . . . . . . . . . . . . . . . . 619

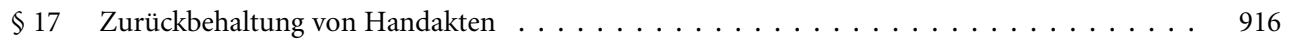

$\$ 18$ Vermittelnde, schlichtende oder mediative Tätigkeit . . . . . . . . . . . . . . 203

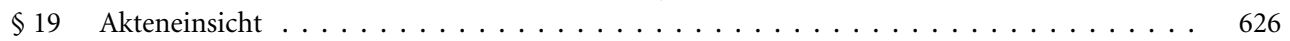

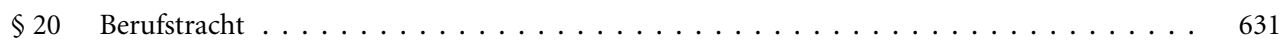


Seite

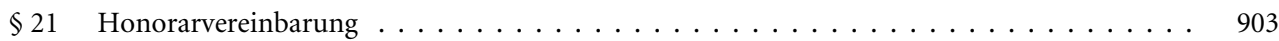

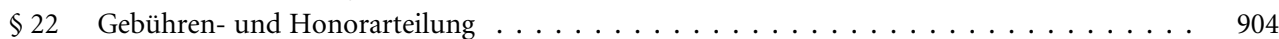

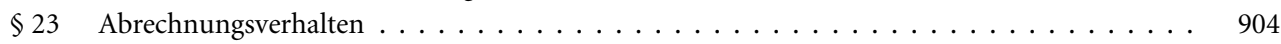

$\$ 24$ Pflichten gegenüber der Rechtsanwaltskammer . . . . . . . . . . . . . . . . . . . . . 1025

$\$ 25$ Beanstandungen gegenüber Kollegen $\ldots \ldots \ldots \ldots \ldots \ldots$

$\$ 26$ Beschäftigung von Rechtsanwälten und anderen Mitarbeitern . . . . . . . . . . . . . . . . . . . . . . . . . . . . . . . . . . . . . . . . . . . . .

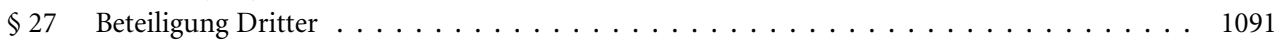

$\$ 28$ Ausbildungsverhältnisse . . . . . . . . . . . . . . . . . . . . . . . . . . . 1041

$\$ 29$ Berufsordnung und CCBE-Berufsregeln . . . . . . . . . . . . . . . . . . . . . . 2179

$\S 29$ a Zwischenanwaltliche Korrespondenz im grenzüberschreitenden

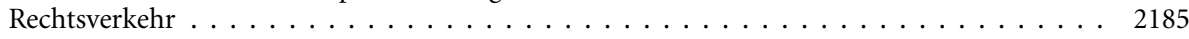

$\S 29 \mathrm{~b}$ Einschaltung eines ausländischen Rechtsanwalts . . . . . . . . . . . . . . . . . . . 2188

$\S 30$ Berufliche Zusammenarbeit mit Angehörigen anderer Berufe . . . . . . . . . . . . . . . . 1092

$\$ 31$ (ehedem Verbot der Sternsozietät, aufgehoben) _ . . . . . . . . . . . . . . . . 1097

$\$ 32$ Beendigung einer gemeinschaftlichen Berufsausübung . . . . . . . . . . . . . . . . . . 1097

$\S 33$ Geltung der Berufsordnung bei beruflicher Zusammenarbeit . . . . . . . . . . . . . . . . 1104

$\$ 34$ Weitere Mitglieder der Rechtsanwaltskammer, ausländische Rechtsanwälte . . . . . . . . . . 2061

\section{Fachanwaltsordnung (FAO)}

Einführung . . . . . . . . . . . . . . . . . . . . . . . . . . . . . . 1923

$\$ 1$ Zugelassene Fachanwaltsbezeichnungen . . . . . . . . . . . . . . . . . . . . . 1930

$\$ 2$ Anforderungen an Kenntnisse und Erfahrungen . . . . . . . . . . . . . . . . . . . . . . . . . . . . . . . . . . . . . . . . . . . .

$\S 3$ Anforderungen an die anwaltliche Tätigkeit . . . . . . . . . . . . . . . . . . . . . . . . . . . . . . . . . . . . . . . . . . .

$\S 4$ Erwerb der besonderen theoretischen Kenntnisse . . . . . . . . . . . . . . . . . . . . . 1939

$\S 4 \mathrm{a} \quad$ Schriftliche Leistungskontrollen . . . . . . . . . . . . . . . . . . . . . . . . . . 1945

$\S 5$ Erwerb der besonderen praktischen Erfahrungen . . . . . . . . . . . . . . . . . . . 1946

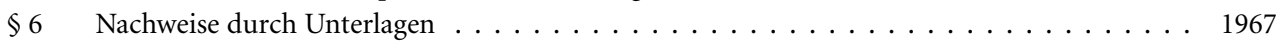

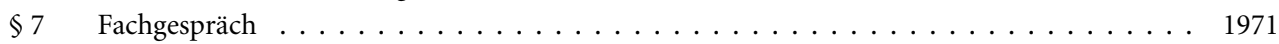

$\S 8 \quad$ Nachzuweisende besondere Kenntnisse im Verwaltungsrecht . . . . . . . . . . . . . . . 1987

$\S 9$ Nachzuweisende besondere Kenntnisse im Steuerrecht . . . . . . . . . . . . . . . . . . . 1988

$\S 10$ Nachzuweisende besondere Kenntnisse im Arbeitsrecht . . . . . . . . . . . . . . . . . . . 1989

$\$ 11$ Nachzuweisende besondere Kenntnisse im Sozialrecht . . . . . . . . . . . . . . . . . . . 1990

$\$ 12$ Nachzuweisende besondere Kenntnisse im Familienrecht . . . . . . . . . . . . . . . . . 1991

$\$ 13$ Nachzuweisende besondere Kenntnisse im Strafrecht . . . . . . . . . . . . . . . . . . . . 1991

$\S 14$ Nachzuweisende besondere Kenntnisse im Insolvenzrecht . . . . . . . . . . . . . . . . . . . 1992

$\$ 14 \mathrm{a}$ Nachzuweisende besondere Kenntnisse im Versicherungsrecht . . . . . . . . . . . . . . . 1993

$\S 14 \mathrm{~b}$ Nachzuweisende besondere Kenntnisse im Medizinrecht . . . . . . . . . . . . . . . . . . . . 1994

$\S 14 \mathrm{c}$ Nachzuweisende besondere Kenntnisse im Miet- und Wohnungseigentumsrecht _ . . . . . 1996

$\S 14 \mathrm{~d}$ Nachzuweisende besondere Kenntnisse im Verkehrsrecht. . . . . . . . . . . . . . . . . . . 1996

$\S 14 \mathrm{e}$ Nachzuweisende besondere Kenntnisse im Bau- und Architektenrecht . . . . . . . . . . . . . 1997

$\S 14 \mathrm{f}$ Nachzuweisende besondere Kenntnisse im Erbrecht . . . . . . . . . . . . . . . . . . . . 1998

$\S 14 \mathrm{~g}$ Nachzuweisende besondere Kenntnisse im Transport- und Speditionsrecht . . . . . . . . . . 1999

$\S 14 \mathrm{~h}$ Nachzuweisende besondere Kenntnisse im gewerblichen Rechtsschutz . . . . . . . . . . . . 2000

$\$ 14 \mathrm{i}$ Nachzuweisende besondere Kenntnisse im Handels- und Gesellschaftsrecht . . . . . . . . . 2000

$\$ 14 \mathrm{j}$ Nachzuweisende Kenntnisse im Urheber- und Medienrecht . . . . . . . . . . . . . . . . . 2001

$\S 14 \mathrm{k}$ Nachzuweisende besondere Kenntnisse im Informationstechnologierecht . . . . . . . . . . . 2002

$\$ 141$ Nachzuweisende besondere Kenntnisse im Bank- und Kapitalmarktrecht . . . . . . . . . . . 2003

$\S 14 \mathrm{~m}$ Nachzuweisende besondere Kenntnisse im Agrarrecht . . . . . . . . . . . . . . . . . . 2004 


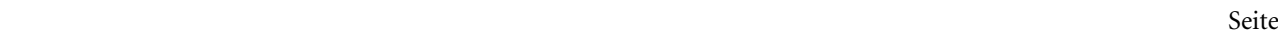

$\S 14 \mathrm{n}$ Nachzuweisende besondere Kenntnisse im internationalen Wirtschaftsrecht . . . . . . . 2005

$\$ 140$ Nachzuweisende besondere Kenntnisse im Vergaberecht . . . . . . . . . . . . . . . . . . 2006

$\$ 14 \mathrm{p}$ Nachzuweisende besondere Kenntnisse im Migrationsrecht . . . . . . . . . . . . . . . . . 2008

$\$ 14 \mathrm{q}$ Nachzuweisende besondere Kenntnisse im Sportrecht . . . . . . . . . . . . . . . . . . 2009

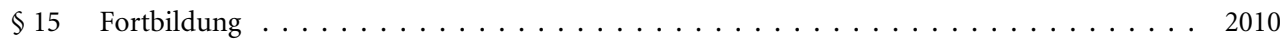

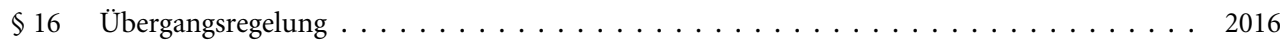

Vorbemerkung Vor $\$ \$ 17 \mathrm{ff} . \ldots \ldots \ldots \ldots \ldots \ldots \ldots$

$\$ 17$ Zusammensetzung der Ausschüsse . . . . . . . . . . . . . . . . . . . . . . . . . . . 2019

$\$ 18$ Gemeinsame Ausschüsse . . . . . . . . . . . . . . . . . . . . . . . . . . 2021

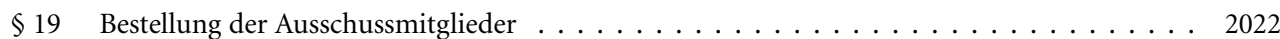

$\$ 20$ Vorzeitiges Ausscheiden aus dem Ausschuss . . . . . . . . . . . . . . . . . . . . . . 2023

$\$ 21$ Entschädigung. . . . . . . . . . . . . . . . . . . . . . . . . . . . . . . . 2024

$\$ 22$ Antragstellung . . . . . . . . . . . . . . . . . . . . . . . . . . . . . . 2024

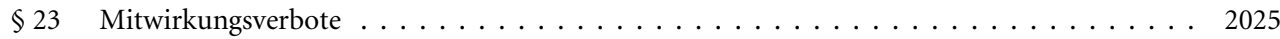

$\$ 24$ Weiteres Verfahren . . . . . . . . . . . . . . . . . . . . . . . . . . . . . 2027

$\$ 25$ Rücknahme und Widerruf . . . . . . . . . . . . . . . . . . . . . . 2034

$\$ 26$ In-Kraft-Treten und Ausfertigung . . . . . . . . . . . . . . . . . . . . . . . 2035

\section{Gesetz über die Tätigkeit europäischer Rechtsanwälte in Deutschland (EuRAG)}

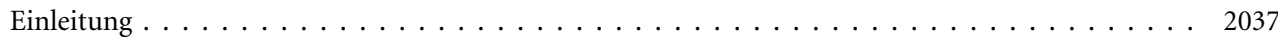

$\S 1$ Persönlicher Anwendungsbereich _ . . . . . . . . . . . . . . . . . . . . 2055

$\S 34$ BORA Weitere Mitglieder der Rechtsanwaltskammer, ausländische Rechtsanwälte . 2061

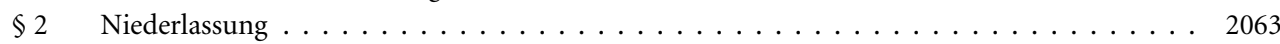

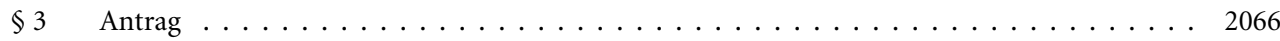

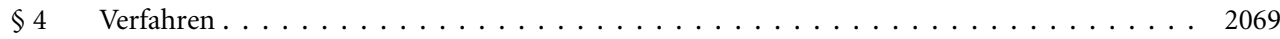

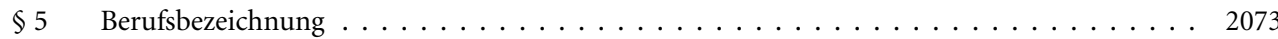

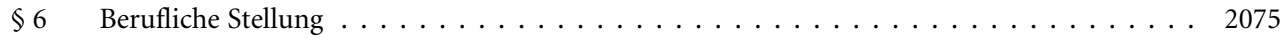

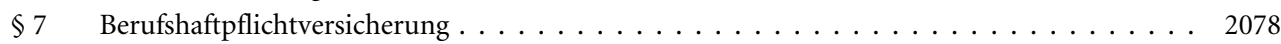

$\$ 8 \quad$ Sozietät im Herkunftsstaat . . . . . . . . . . . . . . . . . . . . . . . . . . 2080

§9 Mitteilungspflichten, rechtliches Gehör . . . . . . . . . . . . . . . . . . . . 2085

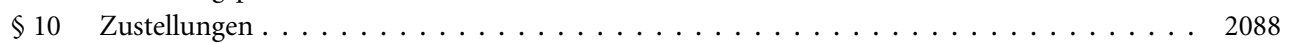

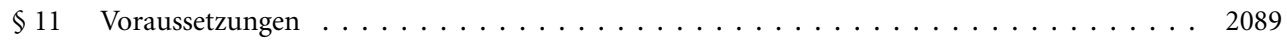

$\$ 12$ Nachweis der Tätigkeit . . . . . . . . . . . . . . . . . . . . . . . . . . . . . . . . . . . . . . . . . . . . . . . . . . . . . . . .

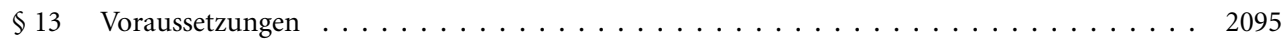

$\$ 14$ Nachweise . . . . . . . . . . . . . . . . . . . . . . . . . . . 2097

$\$ 15$ Gespräch . . . . . . . . . . . . . . . . . . . . . . . . . 2098

$\$ 16$ Antrag auf Feststellung einer gleichwertigen Berufsqualifikation . . . . . . . . . . . . 2099

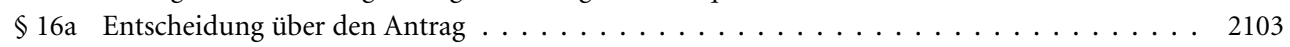

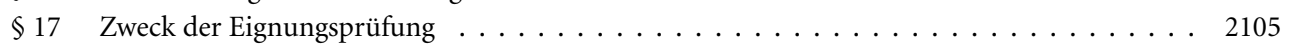

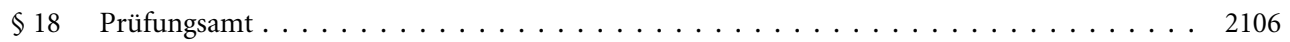

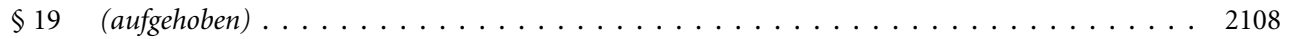

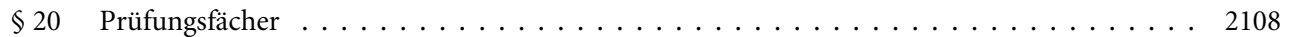

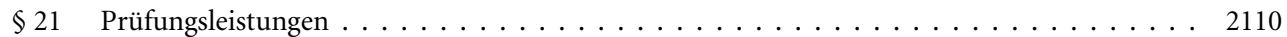

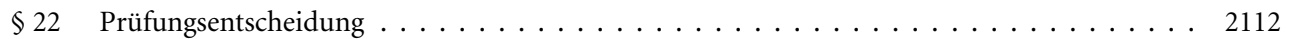

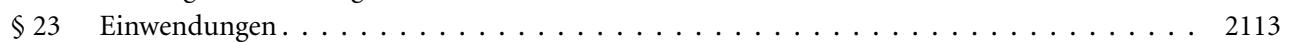

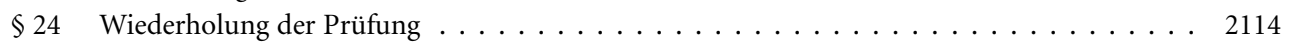

$\$ 25$ Vorübergehende Tätigkeit . . . . . . . . . . . . . . . . . . . . . . . 2115 


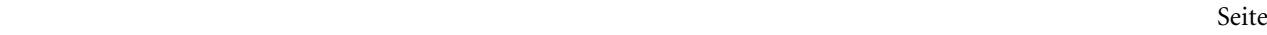

$\S 26$ Berufsbezeichnung, Nachweis der Rechtsanwaltseigenschaft . . . . . . . . . . . . . . 2118

$\$ 27$ Rechte und Pflichten . . . . . . . . . . . . . . . . . . . . . . . . . . . 2119

$\$ 27 \mathrm{a}$ Besonderes elektronisches Anwaltspostfach . . . . . . . . . . . . . . . . . . . . . 2122

$\$ 28$ Vertretung und Verteidigung im Bereich der Rechtspflege . . . . . . . . . . . . . . . . . 2125

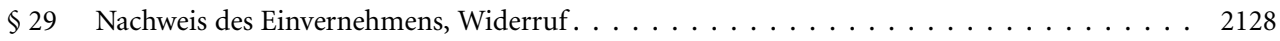

$\$ 30$ Besonderheiten bei Verteidigung . . . . . . . . . . . . . . . . . . . . . . . . . 2129

$\S 31$ Zustellungen in behördlichen und gerichtlichen Verfahren . . . . . . . . . . . . . . . . . 2130

$\S 32$ Aufsicht, zuständige Rechtsanwaltskammer . . . . . . . . . . . . . . . . . . . . . . . . . . . . . . . . . . . . . . . . .

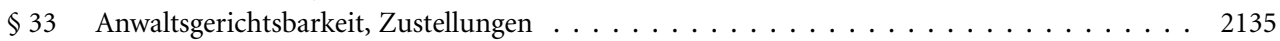

$\$ 34$ Anwaltsgerichtliche Ahndung von Pflichtverletzungen, vorläufige anwaltsgerichtliche

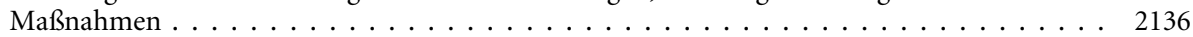

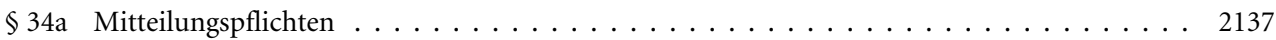

$\S 35$ Rechtsweg in verwaltungsrechtlichen Anwaltssachen . . . . . . . . . . . . . . . . . 2137

$\S 36$ Bescheinigungen des Heimat- oder Herkunftsstaates . . . . . . . . . . . . . . . . . . 2138

$\S 37$ Europäische Verwaltungszusammenarbeit; Bescheinigungen . . . . . . . . . . . . . . . . . 2139

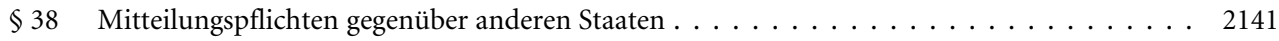

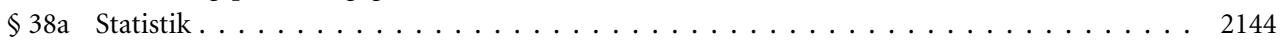

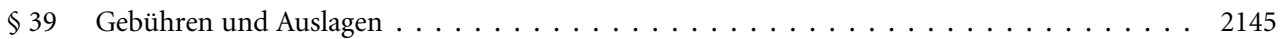

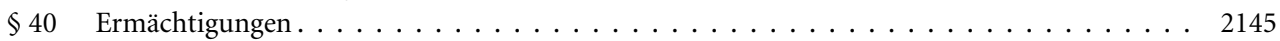

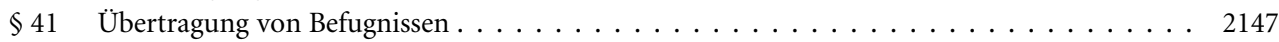

$\S 42$ Anwendung von Vorschriften des Strafgesetzbuches . . . . . . . . . . . . . . . . . . . . . 2148

$\$ 43 \quad$ (aufgehoben) . . . . . . . . . . . . . . . . . . . . . . . . . 2149

Berufsregeln der Rechtsanwälte der Europäischen Union (CCBE) . . . . . . . . . . . . . . . 2151

$\S 29$ BORA Berufsordnung und CCBE-Berufsregeln . . . . . . . . . . . . . 2179

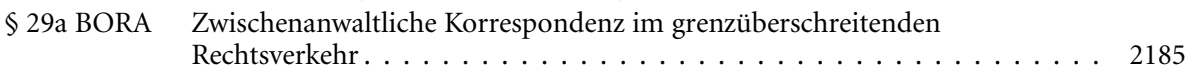

$\S 29$ b BORA Einschaltung eines ausländischen Rechtsanwalts . . . . . . . . . . . 2188

\section{Gesetz über außergerichtliche Rechtsdienstleistungen (RDG)}

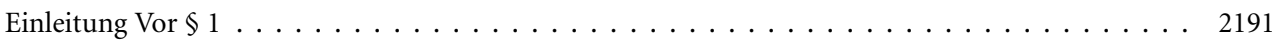

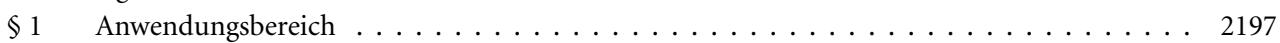

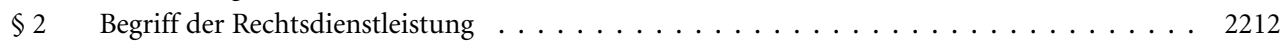

$\$ 3$ Befugnis zur Erbringung außergerichtlicher Rechtsdienstleistungen . . . . . . . . . . . . . 2249

$\$ 4 \quad$ Unvereinbarkeit mit einer anderen Leistungspflicht . . . . . . . . . . . . . . . . . . . . 2256

$\S 5$ Rechtsdienstleistungen im Zusammenhang mit einer anderen Tätigkeit . . . . . . . . . . 2263

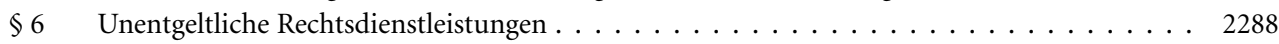

$\$ 7 \quad$ Berufs- und Interessenvereinigungen, Genossenschaften . . . . . . . . . . . . . . . . . . 2298

$\$ 8$ Öffentliche und öffentlich anerkannte Stellen $\ldots \ldots \ldots \ldots \ldots \ldots$

$\$ 9$ Untersagung von Rechtsdienstleistungen . . . . . . . . . . . . . . . . . . . . . 2313

$\$ 10$ Rechtsdienstleistungen aufgrund besonderer Sachkunde . . . . . . . . . . . . . . . . . . . 2317

$\$ 11$ Besondere Sachkunde, Berufsbezeichnungen . . . . . . . . . . . . . . . . . . 2335

$\$ 1$ a Darlegungs- und Informationspflichten bei Inkassodienstleistungen . . . . . . . . . . . . . 2338

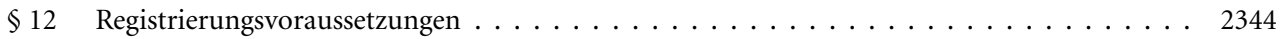

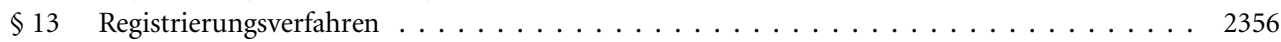

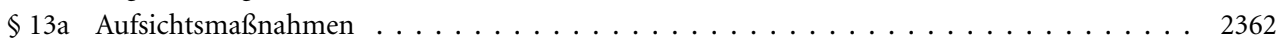

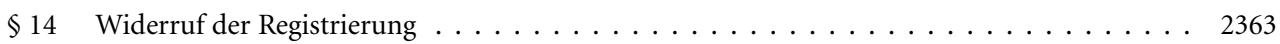


$\begin{aligned} \text { Seite } & \end{aligned}$

$\S 14 \mathrm{a}$ Bestellung eines Abwicklers für Rentenberater . . . . . . . . . . . . . . . . . . . . . . . . . . . . . . . . . . . . . . . . . .

$\$ 15$ Vorübergehende Rechtsdienstleistungen . . . . . . . . . . . . . . . . . . . 2370

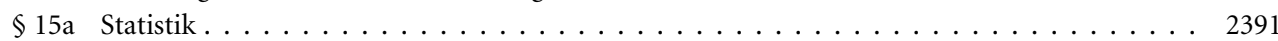

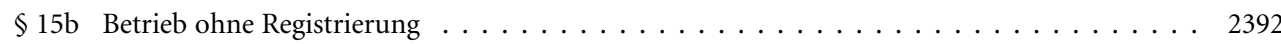

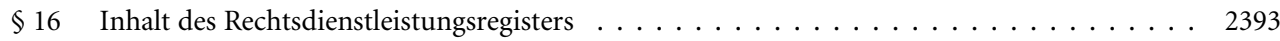

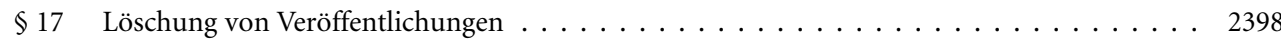

$\$ 18$ Umgang mit personenbezogenen Daten . . . . . . . . . . . . . . . . . . . . . . . . 2402

$\$ 19$ Zuständigkeit und Übertragung von Befugnissen . . . . . . . . . . . . . . . . . . . . . . . 2406

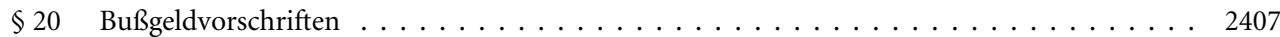

\section{Einführungsgesetz zum Rechtsdienstleistungsgesetz (RDGEG)}

$\$ 1$ Erlaubnisinhaber nach dem Rechtsberatungsgesetz . . . . . . . . . . . . . . . . . . . . 2417

$\$ 2$ Versicherungsberater . . . . . . . . . . . . . . . . . . . . . . . . . 2420

$\$ 3$ Gerichtliche Vertretung . . . . . . . . . . . . . . . . . . . . . . . . . 2421

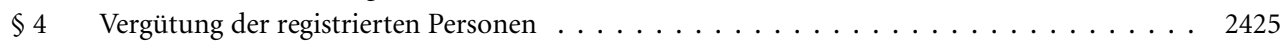

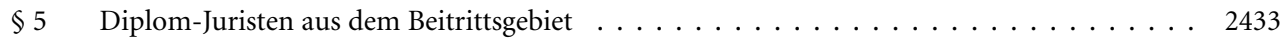

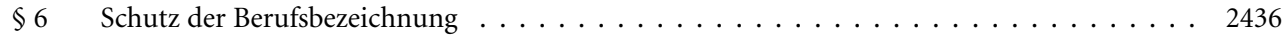

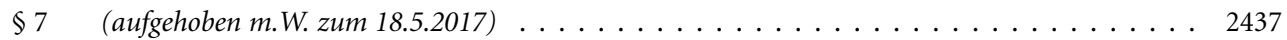

\section{Zivilrechtliche Anwaltshaftung}

A. Überblick . . . . . . . . . . . . . . . . . . . . . . . . . . . . . . . . . . . . 2441

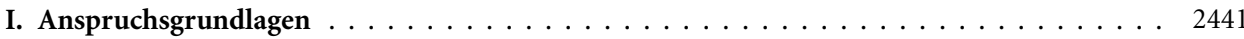

1. Vertragliche Haftung . . . . . . . . . . . . . . . . . . . . . . . . . . 2441

2. Prospekthaftung . . . . . . . . . . . . . . . . . . . . . . . . . . 2456

3. Sonstige gesetzliche Schuldverhältnisse . . . . . . . . . . . . . . . . . . . . . . . . 2458

II. Struktur der Haftung aus dem Anwaltsvertrag . . . . . . . . . . . . . . . . . . . . . 2458

1. Pflichtverletzung . . . . . . . . . . . . . . . . . . . . . . . . . 2458

2. Verschulden . . . . . . . . . . . . . . . . . . . . . . . . 2461

3. Schaden . . . . . . . . . . . . . . . . . . . . . . . . . . . 2462

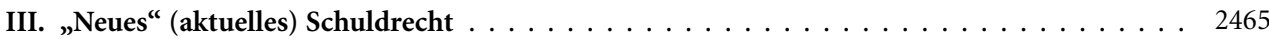

1. Überblick . . . . . . . . . . . . . . . . . . . . . . . . . . . . . 2465

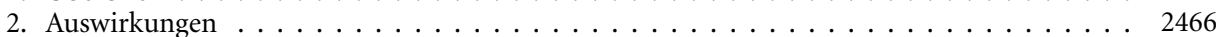

IV. Vertragliche Haftungsbegrenzungen . . . . . . . . . . . . . . . . . . . . . . . . 2470

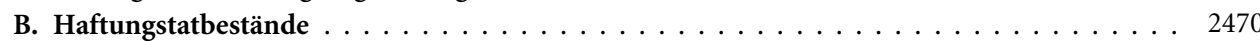

I. Haftung für Fehlverhalten bei der Prozessführung . . . . . . . . . . . . . . . . . . . . . . . 2470

1. Haftungsgrundlage . . . . . . . . . . . . . . . . . . . . . . . . . 2470

2. Haftungsvoraussetzungen . . . . . . . . . . . . . . . . . . . . . . . 2483

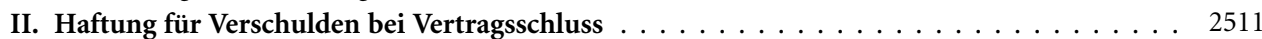

1. Vorvertragliche Aufklärungspflichten . . . . . . . . . . . . . . . . . . . . . . . . 2511

2. Unverzügliche Ablehnung eines Auftrags . . . . . . . . . . . . . . . . . . . . . . . . . . . . . . . . . . . . . . . . . . . . . . . . . . . . .

3. Pflichten nach Scheitern eines Vertragsschlusses . . . . . . . . . . . . . . . . . . . . . . 2514

III. Haftung für Fehlverhalten im Zusammenhang mit Verkaufsprospekten für Kapitalanlagen . 2514

1. Anwalt als Experte . . . . . . . . . . . . . . . . . . . . . . . . . . . 2514

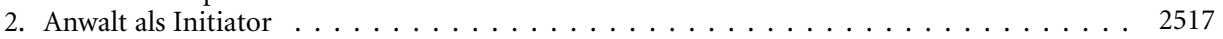

3. Anwalt als Funktionsträger . . . . . . . . . . . . . . . . . . . . . . . . . . . 2519

4. Anwalt als Prospektprüfer . . . . . . . . . . . . . . . . . . . . . . . . . . 2520

5. Deliktische Prospekthaftung des Anwalts . . . . . . . . . . . . . . . . . . . . . . . . 2520

IV. Haftung aus sonstigen gesetzlichen Schuldverhältnissen . . . . . . . . . . . . . . . . 2523

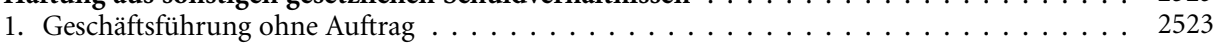

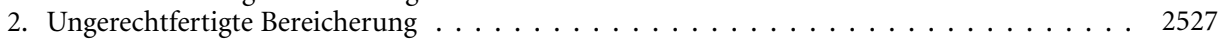

3. Unerlaubte Handlung . . . . . . . . . . . . . . . . . . . . . . . . . . . . . . . . . . 2529 
C. Haftungsadressat . . . . . . . . . . . . . . . . . . . . . . . . . . . . . . . 2540

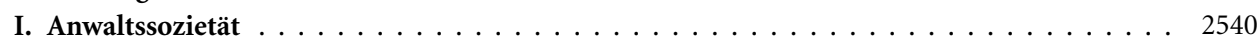

1. Auslegung: Einzel- oder Gesamtmandat . . . . . . . . . . . . . . . . . . . . . . . . . . . . . . 2542

2. Akzessorische Haftung der Sozien auch für berufliche Fehler . . . . . . . . . . . . . . . . . . 2544

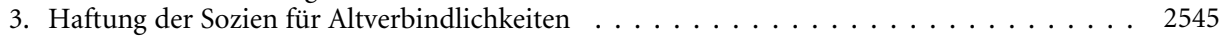

4. Nachhaftung . . . . . . . . . . . . . . . . . . . . . . . . . . . . 2548

5. International tätige Sozietät . . . . . . . . . . . . . . . . . . . . . . 2553

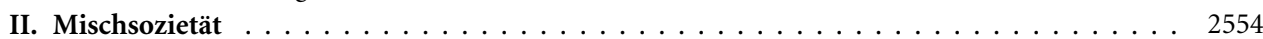

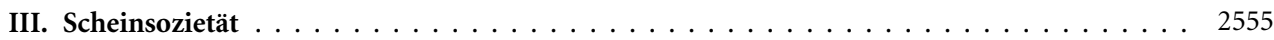

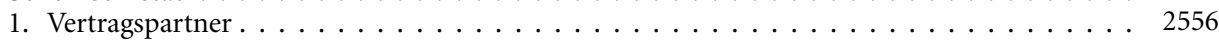

2. Rechtsscheinhaftung . . . . . . . . . . . . . . . . . . . . . . . . 2557

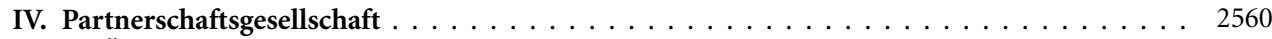

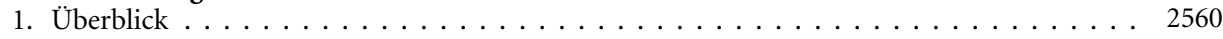

2. Haftungskonzentration . . . . . . . . . . . . . . . . . . . . . . . . 2560

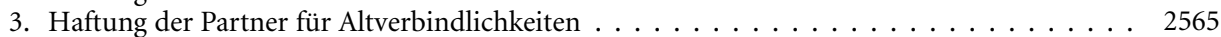

4. Nachhaftung . . . . . . . . . . . . . . . . . . . . . . . . 2566

V. Bürogemeinschaft . . . . . . . . . . . . . . . . . . . . . . . . . . . 2567

VI. Andere Gesellschaften . . . . . . . . . . . . . . . . . . . . . . . . . . . . . . 2568

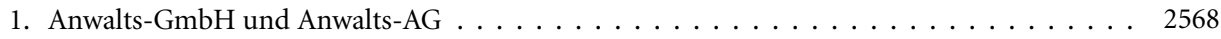

2. Personenhandelsgesellschaften . . . . . . . . . . . . . . . . . . . . . . . . . 2569 . . . . . . . . . . . . . . . . .

3. Gesellschaften ausländischen Rechts . . . . . . . . . . . . . . . . . . . . . . . . 2570

D. Verjährung . . . . . . . . . . . . . . . . . . . . . . . . . . 2571

I. Ansprüche aus vertraglicher Haftung . . . . . . . . . . . . . . . . . . . . . . . 2572

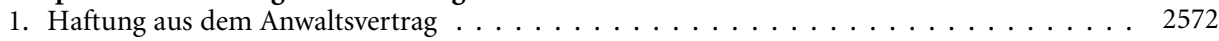

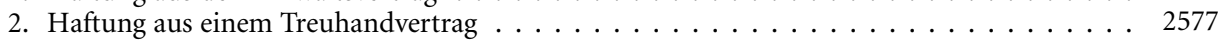

3. Haftung aus einem unechten Anwaltsvertrag . . . . . . . . . . . . . . . . . . . . . . . . . 2577

4. Haftung aus vorvertraglichen Schuldverhältnissen . . . . . . . . . . . . . . . . . . . 2578

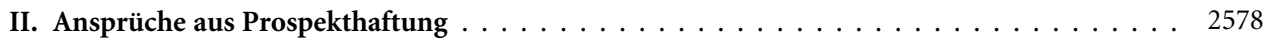

1. Spezialgesetzliche Prospekthaftungsansprüche . . . . . . . . . . . . . . . . . . . 2578

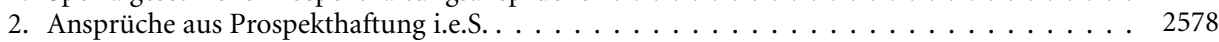

3. Ansprüche aus Prospekthaftung i.w.S. . . . . . . . . . . . . . . . . . . . . . . . 2579

4. Deliktische Prospekthaftungsansprüche . . . . . . . . . . . . . . . . . . . . . . 2580

III. Ansprüche aus sonstigen gesetzlichen Schuldverhältnissen . . . . . . . . . . . . . . . . . . . 2580

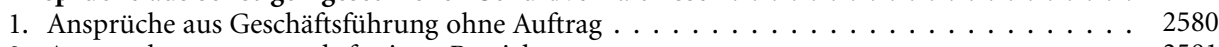

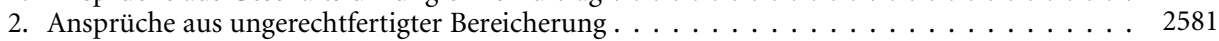

3. Deliktische Ansprüche . . . . . . . . . . . . . . . . . . . . . . . . 2581

\section{Anhänge}

Anhang 1 Satzung der Bundesrechtsanwaltskammer. . . . . . . . . . . . . . . . . . . . 2583

Anhang 2 Verhaltensempfehlungen für Rechtsanwälte im Hinblick auf die Vorschriften des Geldwäschebekämpfungsgesetzes $(\mathrm{GwG})$ und die Geldwäsche, $₫ 261$ StGB . . . . . . . 2591

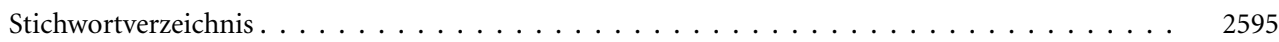

